\title{
The fine spatial structure of methanol masers as an evidence in support of their connection with bipolar outflows
}

\author{
Vyacheslav I. Slysh, Irina E. Val'tts and Sergei V. Kalenskii \\ Astro Space Center, Lebedev Physical Institute, Profsoyuznaya str. \\ 84/32, 117810 Moscow, Russia
}

\begin{abstract}
We studied class I methanol masers in the transition $7_{0}-$ $6_{1} A^{+}$at the frequency $44 \mathrm{GHz}$ with the VLA. The observations on the VLA were made with the angular resolution $0^{\prime \prime} .1$, which was the highest at the moment. It was shown that the masers consist of chains of unresolved spots located on curved lines or arcs. The length of such arcs is from 20 to $1000 \mathrm{AU}$ and the brightness temperature of the strongest masers exceeds $3.6 \times 10^{8} \mathrm{~K}$. The observed location of maser spots is in agreement with their position at the border line between molecular outflows and surrounding molecular clouds. The high brightness temperature implies that the maser condensations have enhanced abundance of methanol due to evaporation of methanol from the surface of dust grains. The mass of maser condensations is less than $4 \times 10^{-5} \mathrm{M} \odot$ and corresponds to planetary masses.
\end{abstract}

\section{Introduction}

Class I methanol masers (most of these masers were detected earlier in our Parkes $44 \mathrm{GHz}$ survey - Slysh et al. 1994) have been studied at $44 \mathrm{GHz}$ with rather high angular resolution in Kogan \& Slysh 1998. Maps of methanol masers with the highest angular resolution were obtained on the BIMA interferometer at frequencies 84 and $95 \mathrm{GHz}$, with a beamwidth of about $5^{\prime \prime}$ (Batrla \& Menten 1998, Plambeck \& Menten 1990, Pratap \& Menten 1992). The masers were shown to consist of several unresolved spots, which do not coincide with known objects. Now we report the results of new VLA observations of class I methanol masers in the 44-GHz transition, with an angular resolution of $0^{\prime \prime} .1$ and studying the structures of the masers based on the VLA maps.

\section{Observations and data reduction}

Observations of the 44-GHz methanol line were carried out on June 28, 1998, for five sources (M8E, W33Met, L379IRS3, GGD27 and G14.33-0.64). The VLA (NRAO, USA) was in the AB configuration. We used eleven of 27 antennas that were equipped with $7-\mathrm{mm}$ receivers. The synthesized beamwidth of the interferometer was from $0^{\prime \prime} .1 \times 0^{\prime \prime} .25$ to $0^{\prime \prime} .1 \times 0^{\prime \prime} .17$ (depending on the direction toward the source). The sources were observed with a bandwidth of $3125 \mathrm{kHz}$, 

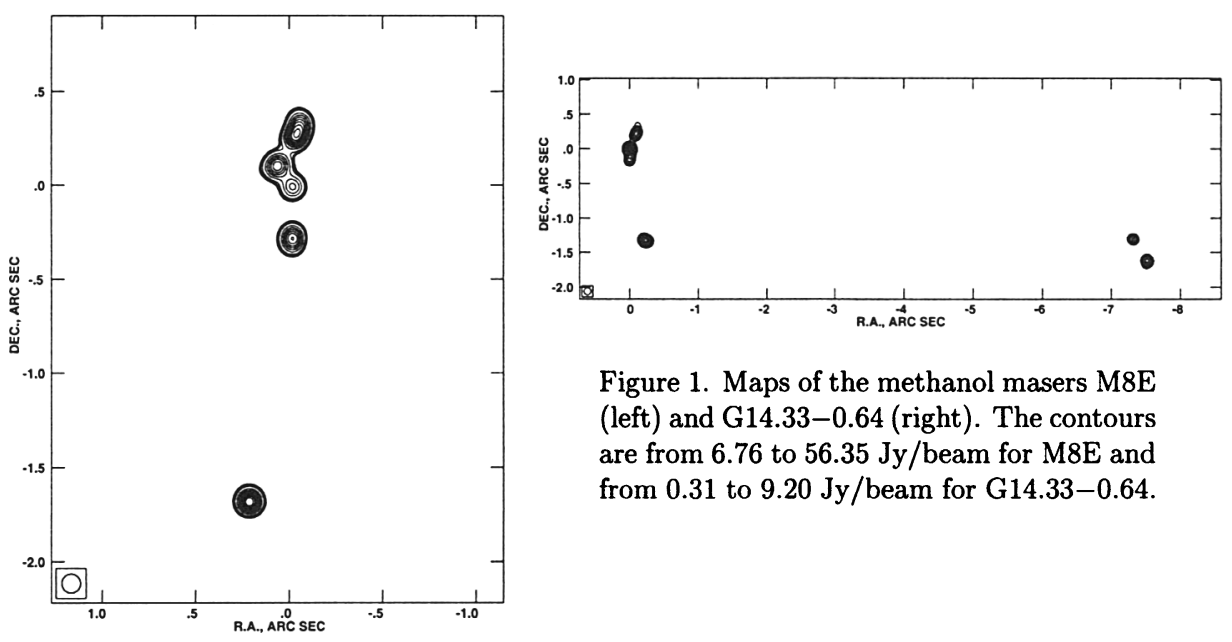

Figure 1. Maps of the methanol masers M8E (left) and G14.33-0.64 (right). The contours are from 6.76 to $56.35 \mathrm{Jy} /$ beam for M8E and from 0.31 to $9.20 \mathrm{Jy} /$ beam for G14.33-0.64.

so that the total velocity coverage was $23.3 \mathrm{~km} / \mathrm{s}$. 128 channels were recorded, yielding a velocity resolution of $0.17 \mathrm{~km} / \mathrm{s}$. M8E and GGD27 were observed with a higher spectral resolution of $0.042 \mathrm{~km} / \mathrm{s}$. Fig. 1 shows representative maps of two masers: M8E and G14.33-0.64. The full description of maser maps is given in the paper of Slysh et al. 1999.

\section{Discussion}

Mapping class I methanol masers with an angular resolution of $0^{\prime \prime} .1$ has allowed us to reveal their structure. In most cases, the masers form chains or clusters of maser spots. The spots themselves are unresolved with the VLA beam, and the upper limits of the size is typically $0^{\prime \prime} .05$. The corresponding lower limit on the brightness temperatures for various sources is between $5.7 \times 10^{6}$ and $3.6 \times 10^{8} \mathrm{~K}$. The chains of spots visible in the maps for most of the masers may trace the geometry of the maser emission regions. One possibility is that the maser spots are bright knots on long filaments (up to $10000 \mathrm{AU}$ ) with thickness of less than $200 \mathrm{AU}$, which could form in regions of intersection of two spherical shock waves, possibly arising during collisions of molecular outflows. In another model in which the chains of maser spots are located on the spherical surface of a shock wave, the maser spots are observed at the tangential surface of the shock front, where the amplification path is at maximum. The projected sizes of the maser spots can be much smaller than their sizes along the line of sight. In this case, the maser spots outline the outer contours of outflows. None of the class I methanol maser spots display the high velocities observed in molecular outflows. Their radial velocities are usually at the center of the thermal line, which forms in the medium surrounding the molecular cloud, and the velocity dispersion is always smaller than the width of the thermal line. If the maser spots are located at the tangential surface of a shock wave, they can still move with high velocities, but only in the transverse direction. Since the amplification factor 
in the tangential direction is greater than in the transverse direction, the maser emission will be observed with a small line-of-sight velocity. In this picture, the maser condensations move with the shock surface, but the only ones that are visible are those that move in the transverse direction. This model can be tested by deriving the tangential velocities from the proper motions of the maser spots. The tangential-velocity estimates in W33Met and L379IRS3 that we have obtained here are not sufficiently precise to detect proper motions with values typical for molecular outflows or shock waves. The upper limits on the transverse velocities are 30 and $60 \mathrm{~km} / \mathrm{s}$ for L379IRS3 and W33Met, respectively, which are larger than the typical velocities of molecular outflows $(20 \mathrm{~km} / \mathrm{s})$. More precise proper-motion measurements are required to test the propagating shock-wave model for the maser spots. On the other hand, the maser condensations could be stationary, while the shock flows around them. In this picture, the interaction with the shock increases the temperature, methanol evaporates from the surfaces of dust grains, and the methanol abundance increases in the condensations to a value sufficient for the generation of maser emission. After the passage of the shock, the condensations remain stationary. The origin of small (100-200 AU) maser condensations is unclear. One possibility is that they appear as a result of Rayleigh-Taylor instabilities that arise during the interaction of a shock with the surrounding medium. However, we do not have enough observational evidence to confirm any specific model.

Though the connection of methanol masers with bipolar outflows is well established, there are some masers (for example, NGC6334-I(N)) in which outflows are not observed. In addition, in many cases, emission from excited vibrational levels in the $\mathrm{H}_{2}$ molecule, which is a good tracer of interactions between an outflow and the surrounding medium, is not observed. If maser spots are stationary condensations in the surrounding medium with a molecular outflow flowing around them, we might expect a correspondence between maps of the methanol masers and of the $\mathrm{H}_{2}$ line emission. For the sources we have considered here, we found no such correspondence. If the observed projected sizes of the maser condensations 100-200 AU are equal to their line-of-sight sizes, we can estimate their masses, assuming their density is that of molecular hydrogen, $10^{6} \mathrm{~cm}^{-3}$ (as follows from the maser-pumping model (Menten et al. 1998)): $8 \times 10^{28} \mathrm{~g}$, or $4 \times 10^{-5} \mathrm{M} \odot$. Such small condensations would not be detected in thermal molecular lines, and only the maser emission would make them visible.

The lower limit on the brightness temperature, $3.6 \times 10^{8} \mathrm{~K}$, corresponds to a maser optical depth of the order of -15 , and to a methanol column density of the order of $10^{16} \mathrm{~cm}^{-2}$ (Liechti \& Walmsley 1997). If the linear size of a maser condensation is $200 \mathrm{AU}$ and the $\mathrm{H}_{2}$ density is $10^{6} \mathrm{~cm}^{-3}$, the methanol abundance should be of the order of $3 \times 10^{-6}$. This is three to four orders of magnitude greater than in the interstellar medium. Such a strongly enhanced methanol abundance could be a consequence of evaporation of methanol from the surface of dust grains, stimulated by heating from a shock wave. If the maser condensations are extended along the line of sight, as in our model where they are localized on the tangential surface of a shock wave, their line-of-sight sizes may be much greater than their projected sizes, and the corresponding estimated masses will be considerably higher. 


\section{Conclusions}

We have studied the structure of class I methanol masers based on our highresolution VLA maps. A typical pattern formed by these masers is a chain of bright spots located along arclike curves with length of several thousands AU. The spots themselves are not resolved and have sizes less than several hundred AU. Some sources have several maser spot groups separated by few tenths of a parsec. The methanol maser spots have no counterparts in $\mathrm{OH}$ or $\mathrm{H}_{2} \mathrm{O}$ masers and do not coincide with radio continuum or infrared sources. At the same time, class I methanol masers are tightly connected with molecular outflows. Our results support the hypothesis that the methanol masers arise in zones of interaction of molecular outflows between themselves or with the surrounding medium. Proceeding from the fact that the brightness temperatures of these masers are very high, more than $10^{8} \mathrm{~K}$, we conclude that the maser condensations should have strongly enhanced methanol abundance as a result of methanol evaporation from the surface of dust grains. The masses of the maser condensations do not exceed $4 \times 10^{-5} \mathrm{M}_{\odot}$, roughly corresponding to planetary masses.

Acknowledgments. The authors are grateful to the VLA staff for the help with the observations. The work was done under a partial financial support from the INTAS (grant No. 97-11451) and the Russian Foundation for Basic Research (grant No. 01-02-16902).

\section{References}

Batrla, W. \& Menten, K.M. 1998, ApJ, 329, L117

Kogan, L.R. \& Slysh, V.I. 1998, ApJ, 497, 800

Liechti, S. \& Walmsley, C.M. 1997, A\&A, 321, 625

Menten, K.M., Walmsley, S.M., Henkel, C., \& Wilson, T.L. 1998, A\&A, 198, 253

Plambeck, R.L. \& Menten, K.M. 1990, ApJ, 364, 555

Pratap, P. \& Menten, K.M. 1992, Proc. Conf. Astrophysical Masers, Clegg, A. \& Nedoluha. G., Eds., Lect. Notes. Phys., Vol. 103, p. 211 (SpringerVerlag: Heidelberg)

Slysh, V.I., Kalensky, S.V., Val'tts, I.E., \& Otrupcek, R. 1994, MNRAS, 268, 464

Slysh, V.I., Val'tts, I.E., Kalenskii, S.V., \& Golubev, V.V. 1999, Astronomy Reports, 43, 785 